\title{
Using Dynamic Time Warping of T0 Contours in the Evaluation of Cycle-to-Cycle Pitch Detection Algorithms
}

\author{
Carlos Ferrer, Diana Torres, and María E. Hernández-Díaz \\ Center for Studies on Electronics and Information Technologies, Central University of Las \\ Villas, C. Camajuaní, km 51ำ, Santa Clara, CP 54830, Cuba \\ \{cferrer, dtb, mariae\} @uclv.edu.cu
}

\begin{abstract}
This paper addresses the comparison of Pitch Detection Algorithms working on a cycle to cycle basis. An alignment problem between detected and reference pitch contours is described and a Dynamic Time Warping procedure to correct it is proposed. The method is evaluated using hand-marked real signals and three well known Pitch Detection Algorithms. Results demonstrate the occurrence of shifts in practice and the usefulness of the proposed Dynamic Time Warping procedure.
\end{abstract}

Keywords: Dynamic time warping, pitch determination, waveform matching.

\section{Introduction}

Pitch (T0) determination is one of the most common and needed tasks in speech processing. The requirements of each specific application (e.g. speech coding in communications systems, prosodic and intonation analysis in phonetics, or perturbation measurements in clinical studies) are different, causing the proliferation of a large number on Pitch Determination Algorithms (PDAs). The most extensive work in categorization and compilation of PDAs is the one by Hess ([1], later updated in [2]). The number of available PDAs continues to grow each year (e.g. Interspeech-2007 dedicated 2 sessions to pitch extraction) due to the absence of a definitive reliable and accurate solution for the different applications. The evaluation and comparison of the performances of this large number of PDAs results of paramount importance. However, there is no standard comparison procedure for this task.

This paper addresses the latter topic, in the group of PDAs capable of tracking cycle-to-cycle T0 contours, specifically oriented to pathologic voices. The remaining of this section describes some methodological aspects of cycle-to-cycle PDAs and its comparison, revealing an unreported inconsistency of some measures.

\subsection{Cycle-to-Cycle Pitch Determination}

PDAs can be broadly categorized in "short-term" PDAs (working on "small" analysis frames where signal can be considered stationary and contain several pitch pulses, but yielding a single estimate of T0 for the whole frame) and cycle-to-cycle PDAs. The latter PDAs work over even smaller frames and provide individual pitch markers, and 
hence, a more detailed T0 contour. This detailed $\mathrm{T} 0$ contour is required to obtain most of the time-domain perturbation measures used in Vocal Quality assessment (i.e. jitter, shimmer, and many forms of harmonics-to-noise ratios).

Any types of cycle-to-cycle PDA can provide the necessary T0 contour for the calculation of perturbation measures in clinical applications. However, there is a general consensus ([3],[4],[5],[6],[7]) in that Waveform Matching (WM) techniques are the most precise T0 estimators. An exception to the reported overall superior performance of WM techniques is found in [8] but for very broad search ranges $(40-800 \mathrm{~Hz})$ and out of the scope of perturbation measures. In addition, many cycle-to-cycle PDAs work "refining" an existing T0 estimate or "mean" $\left(\mathrm{TO}_{\mathrm{E}}\right)$, known in synthetic signals, and obtainable by different means in real signals (e.g. manually by visual inspection in [9], from a low pass filtered version of the signal in [3] or using a short-term PDA as in the Praat system [10]). The existence of such T0 estimate allows the use of narrow search ranges $\left(S_{r}\right)$, avoiding the occurrence of some of the errors more accounted for in several studies, like doubling or halving the actual T0.

\subsection{Measures of Performance}

The performance of the PDAs is measured according to the similitude between the detected T0 contour $\left(T_{d}(n)\right)$ and a reference contour $\left(T_{r}(n)\right)$. When comparing shortterm PDAs, " $n$ " is the frame index, while in cycle-to-cycle PDAs it stands for the pulse index. A brief survey of representative measures of performance follows. For practical purposes, measures are grouped in two categories: "reliability" measures, which account for how frequently the PDA misses the right T0, and "accuracy" measures, accounting for the magnitude of the differences between $T_{d}(n)$ and $T_{r}(n)$.

\subsubsection{Reliability Measures}

Voicing Determination: Subdivided into Voiced/Unvoiced (V/U) and Unvoiced/Voiced (U/V) errors ([11][4][8][7]). Mostly relevant for short term PDAs, can be excluded from the analysis in perturbation measures, where voicing is a prerequisite and can be determined by other means.

Gross Errors (GE): Number of times the difference among the two contours is greater than a certain threshold $t_{h}$. Most of the time the threshold is normalized to $T_{r}(n)$ and given in percent (5\% in [12], 20\% in [4] and [8]), although absolute values have also been used (5 samples in [17], $1 \mathrm{~ms}$ in [11] and [13], $10 \mathrm{~Hz}$ in [14]).

Pitch Insertions and Deletions (PI/PD): Sometimes included in GE (in [8] GE is further divided into "too low" and "too high", and "too low" errors were linked to the detection of subharmonics). In [7] the occurrence of PI and PD was connected to the detection of multiples and submultiples, respectively, of the true T0 $\left(T_{r}(n)\right)$. In [16] a change (increment or reduction) in the number of detected pulses is also linked to the detection of multiples and submultiples of $T_{r}(n)$, whenever $T_{d}(n)$ falls outside of the range $0.75 T_{r}(n)<T_{d}(n)<1.5 T_{r}(n)$. In perturbation measures, if the estimate of $T_{r}$ is available $\left(\mathrm{TO}_{\mathrm{E}}\right.$ ), it is be possible to limit $S_{r}$ (the search range of $T_{d}$ ), and this type of errors would not occur. $S_{r}$ is usually symmetrical to $\mathrm{T} 0_{\mathrm{E}}$ and given relative to it, establishing that $\mathrm{T}_{\mathrm{E}}\left(1-S_{r}\right)<T_{d}(n)<\mathrm{T} 0_{\mathrm{E}}\left(1+S_{r}\right)$. Different values for $S_{r}$ have been used: $10 \%$ in [9] and [23], 5-15\% in [3], 15\% in [15], 20\% in [10]. The symmetrical limit to $S_{r}$ 
avoiding the detection of multiples and submultiples of T0 is $33 \%$ (i.e. being the maximum less than twice the minimum), an option to the asymmetrical range in [16].

\subsubsection{Accuracy Measures}

Root Mean Squared (RMS) measures of Fine Errors: Used as the complementary measure to GE, measures the mean squared value of the differences among $T_{d}(n)$ and $T_{r}(n)$, when the differences are smaller than the same threshold $t_{h}$ used to determine GE. Slight differences exist across studies, e.g. the RMS is most of the times calculated using the absolute differences ([17][14][4][13]) in milliseconds, but also normalizing the differences by the $T_{r}(n)$ value [6] and removing the mean error [11].

Jitter Values: The values of jitter for the reference and detected contours can be compared, and the difference would yield an estimate of the PDA's success. There are many formulas to account for jitter (see [18] for some variants), although a representative expression is given by (1), the first order perturbation:

$$
\alpha_{d}=\frac{1}{N-1} \sum_{n=1}^{N-1} \frac{\left|T_{d}(n+1)-T_{d}(n)\right|}{0.5^{*}\left(T_{d}(n+1)+T_{d}(n)\right)} * 100
$$

which is the one used in [3] and [6]. The usefulness of $\Delta \alpha=\left|\alpha_{d}-\alpha_{r}\right|$ is criticized in [6] by noting that two constant but different $T_{d}(n)$ and $T_{r}(n)$ would yield the same value of $\alpha_{d}=\alpha_{r}=0$, thus giving a perfect coincidence in spite of being the contours different. The criticism can actually be extended: there are infinite pairs of random contours $T_{d}(n)$ and $T_{r}(n)$ which yields coincident $\alpha_{\mathrm{d}}$ and $\alpha_{\mathrm{r}}$ of any value, not only zero. In spite of this inconsistency, perturbation measures like $\alpha$ form the core of the reported comparisons among the PDAs of commercial systems [19][20][21][22][16].

Average Normalized Difference: Non-RMS measures of the difference between $T_{d}(n)$ and $T_{r}(n)$ have also been proposed [23]. An expression close to the definition of " $\alpha$ " in (1) which can be interpreted as the "jitter" introduced by the PDA (and named " $\beta$ " following the suggestion in [6] as a necessary complement to " $\alpha$ ") is:

$$
\beta=\frac{1}{N} \sum_{n=1}^{N} \frac{\left|T_{d}(n)-T_{r}(n)\right|}{T_{r}(n)} * 100
$$

Only measures considering differences between $T_{r}(n)$ and $T_{d}(n)$ (like " $\beta$ " or the ones in the RMS group) can reveal the accuracy of a PDA. The use of measures like $\Delta \alpha$ is justified only when $T_{d}(n)$ and $T_{r}(n)$ are not available to the researcher.

\subsection{Non Reported Failure of Existing Measures of Performance}

The existing set of measures of performance and comparison procedures do not account for the occurrence of shifts in the $T_{d}$ contour. Figure 1 shows a segment comprising pulses 25-36 of $T_{d}(n)$ (circles) and $T_{r}(n)$ (squares) for a given signal. In terms of the difference between $T_{d}(n)$ and $T_{r}(n)$ and using a $t_{h}=10 \%$ there are six GE in the interval (pulses 28-33). However, five out of these six "erroneous" $T_{d}(n)$ correspond to $T_{r}(n+1)$. In terms of perturbation measures, $T_{d}(n)$ follows the "true" variability of $T_{r}(n)$ with the exception of two GE, the one in pulse 28 (which "shifted" the contours) and the one in pulse 33 , which by coincidence restored the alignment. 


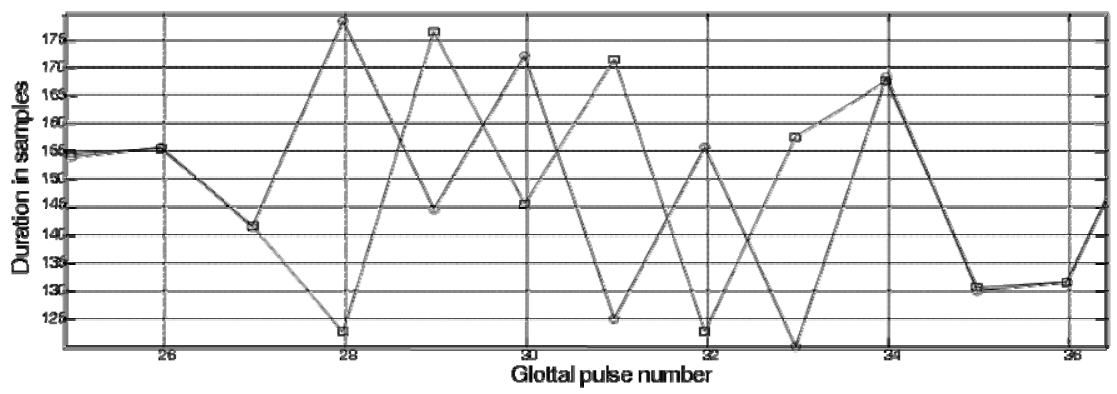

Fig. 1. Example of shift between $T_{d}(n)$ (circles) and $T_{r}(n)$ (squares)

It must be noted that no PD occurred in $T_{d}(n)$ to make $T_{d}(27)=T_{r}(27)$ and $T_{d}(28)=T_{r}(29)$. No PI occurred either in the realignment error in pulse \#33. The number of pitch markers is not changed by these "shifting" errors. Shifting errors are a non-reported type of errors, which may be included in GE for their magnitude but, unlike "normal" GE, require additional steps in aligning the compared contours. It must also be noted that, even with a narrow $S_{r}$ avoiding the detection of multiples and submultiples, PIs and PDs can actually occur. Cumulative errors in the same direction can cause an actual difference in the number of pitch marks in the two contours. These PIs and PDs are different from the ones described in [7] and [16] since they do not occur in the time-span of a single pulse (which is not possible due to the $S_{r}$ limit), but over several pulses. Previous works using narrow $S_{r}$ have also overlooked the possibility of occurrence of "cumulative" PIs and PDs.

\section{Time Warping of $T_{d}$ and $T_{r}$}

To account for the errors described in section 1.3. and allow the comparison of $T_{d}(n)$ and $T_{r}(n)$, both contours should be aligned with respect to the corresponding pulse indexes. This can be performed by means of a dynamic time warping (DTW) procedure similar to the ones used in speech recognition systems.

We define the time series of pitch markers positions $P_{d}\left(n_{d}\right)$ and $P_{r}\left(n_{r}\right)$ such that $T_{d}\left(n_{d}\right)=P_{d}\left(n_{d}+1\right)-P_{d}\left(n_{d}\right)$ and $T_{r}\left(n_{r}\right)=P_{r}\left(n_{r}+1\right)-P_{r}\left(n_{r}\right)$. Note that pulse indexes for the detected and reference contours have been separated in $n_{d}$ and $n_{r}$, respectively. The knowledge of $P_{d}\left(n_{d}\right)$ and $P_{r}\left(n_{r}\right)$ allows the setting of relevant index constraints to the time warping algorithm.

For notation brevity, we define a one-point pitch contour distance function $D(a, b)$ as $D(a, b)=\left|T_{d}\left(n_{d}+a\right)-T_{r}\left(n_{r}+b\right)\right|$. This way, a Boolean condition for the occurrence of a $\mathrm{GE}$ for a given position in $n_{d}$ and $n_{r}$ is $G E_{c o n d}=\left(D(0,0)>t_{h}\right)$. The condition for an apparent need of shifting $T_{d}$ to the left can be written, checking two points:

$S L_{\text {cond }}=\left(D(0,-1)<t_{h}\right) A N D\left(D(1,0)<t_{h}\right) A N D\left(P_{r}\left(n_{r}\right)>P_{d}\left(n_{d}\right)\right)$

The check for two pulse lengths coincidences is performed to increase the certainty that a correction is needed. Comparing more than two pulses can have negative effects, since all the compared pulses should maintain the same shift and no GE occur. The additional check regarding the positions of the current couple of markers 
$\left(P_{r}\left(n_{r}\right)>P_{d}\left(n_{d}\right)\right)$ is included to guarantee that their time positions are coherent with the apparent required shift.

A need of a correction to the right can similarly be tested by:

$S R_{\text {cond }}=\left(D(0,1)<t_{h}\right)$ AND $\left(D(-1,0)<t_{h}\right)$ AND $\left(P_{r}\left(n_{r}\right)<P_{d}\left(n_{d}\right)\right)$

A shift of $T_{d}$ to the right with respect to $T_{r}$ can be performed by decrementing $n_{d}$ to $n_{d}-1$, while the shift of the contour to the left would be achieved with an increment. However, we preferred to perform the "left shift" of $T_{d}$ by actually reversing $T_{r}$ (setting $\left.n_{r}=n_{r}-1\right)$. Making the corrections always backwards allows the alignment of a larger number of contour points.

We perform the correction of shifts due to actual changes in the number of detected pulses (occurrence of PIs or PDs) whenever:

$$
\begin{aligned}
& P D_{\text {cond }}=P_{d}\left(n_{d}-1\right) \geq P_{r}\left(n_{r}+1\right) \\
& P I_{\text {cond }}=P_{d}\left(n_{d}+1\right) \leq P_{r}\left(n_{r^{-}}-1\right)
\end{aligned}
$$

The alignment procedure proposed in this work is depicted in the flowchart in Figure 2.

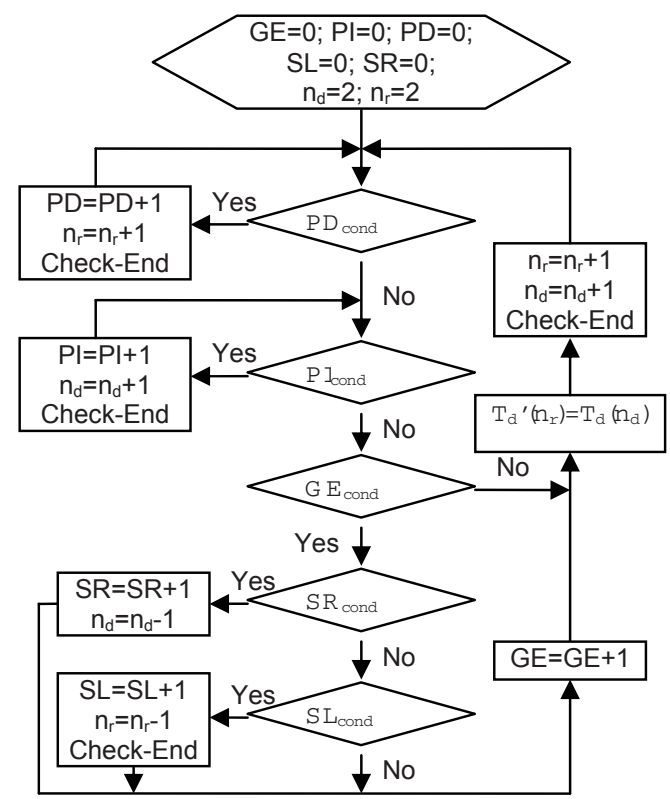

Fig. 2. Flowchart of the alignment procedure devised

As a result of the algorithm, an "aligned" detected contour $\left(T_{d}{ }^{\prime}(n)\right)$ is obtained, comparable pulse by pulse to the reference contour $T_{r}(n)$. The number of GE, PD, PI, SL and SR (shifts to left and right, respectively) is also reported. When the algorithm ends (by reaching the limit of pulses in either $T_{d}$ or $T_{r}$ ) the $T_{d}$ ' contour can be compared to $T_{r}$ in the same pulse indexes. 


\section{Experiments}

The proposed comparison procedure was evaluated by testing three well known WM variants (the one in [9], its modification in [3] and the super-resolution method in [23]) against handmarked real signals. These WM PDAs have shown better performance than other cycle-to-cycle [3] and short term PDAs [4][6][7], but a direct comparison among them has not been performed. To evaluate the actual occurrence in real signals of the different types of shifts described, samples were taken from the Massachussets Eye and Ear Infirmary / Kay Elemetrics Database [24]. Since the database is not annotated, handmarking of individual pitch epochs was required. To save time, a screening procedure was applied, consisting of several steps, to select a reduced set of signals (namely a dozen) which should result representative for our purposes. The steps of the screening procedure, and their results, are the following:

$\mathbf{1}^{\text {st }}$ : Evaluate only pathological samples. This reduces the number of signals from 710 to 657 .

$\mathbf{2}^{\text {nd }}$ : Use signals where at least one of the considered methods fails. To guarantee this failure, an unsupervised run (unchecked $\mathrm{T} 0_{\mathrm{E}}$ estimate) of the three methods was performed. The median of the resulting T0 from the classic AMDF short-term PDA [25], with search range from 80 to $600 \mathrm{~Hz}$, was used as the $\mathrm{T} 0_{\mathrm{E}}$ for the $\mathrm{WM}$ methods. Signals where non-significant differences (12\%) were found among the WM methods were discarded. This reduced the number of signals to consider to 108 .

$3^{\text {rd }}$ : Spectrographic check: The spectrogram of the 108 signals were inspected to suppress signals where the $\mathrm{AMDF} T 0_{\mathrm{E}}$ estimate resulted wrong (15) and completely aperiodic signals (58). This step left 39 signals to consider.

$4^{\text {th }}$ : Oscilographic check: Two factors further reduced the number of signals when visually inspected in the time domain. 15 signals showed a time-varying pattern that made impossible to uniquely define the pitch marker to use. From the remaining 24 signals, equal numbers were more adequately marked by the main positive peak (12) and by the negative peak (12). To make the handmarking process more uniform, it was decided to use only the signals which favored the positive peak markers.

The hand marking process produced 1597 pitch markers (average F0=133 Hz).

\section{Results}

The alignment procedure was performed by setting the GE threshold to $3 \%$ of the estimated mean period $\left(t_{h}=0.03 \mathrm{~T} 0_{\mathrm{E}}\right)$. This is somewhat more strict than values previously reported (5-20\%) but this threshold is also used for checking the $\mathrm{SL}_{\text {cond }}$ and $\mathrm{SR}_{\text {cond }}$, and lower values prevent unnecessary adjustments. In addition to the reliability measures produced by the alignment procedure (GE, PI, PD, SR and SL), the values for the accuracy measures $\alpha$ and $\beta$ were also calculated. To better appraise the effect of the alignment procedure, $\alpha$ and $\beta$ were obtained for the contours before and after the alignment. PDAs methods are denoted Mil [9], Tit [3], and Med [23]. 
Table 1. Results of the DTW procedure for the three PDAs tested

\begin{tabular}{lccccccccc}
\hline & \multicolumn{3}{c}{ Accuracy Measures } & \multicolumn{5}{c}{ Reliability Measures } \\
\cline { 2 - 11 } & $\left|\alpha_{\mathrm{d}}-\alpha_{\mathrm{r}}\right|$ & $\left|\alpha_{\mathrm{d}}{ }^{\prime}-\alpha_{\mathrm{r}}{ }^{\prime}\right|$ & $\beta$ & $\beta$ & GE & SR & SI & PI & PD \\
\hline Mil & 0.45 & 0.41 & 3.19 & 1.43 & 164 & 14 & 14 & 2 & - \\
\hline Tit & 0.39 & 0.40 & 2.71 & 1.31 & 139 & 15 & 10 & 1 & - \\
\hline Med & 0.40 & 0.40 & 2.82 & 1.32 & 123 & 10 & 11 & 1 & - \\
\hline
\end{tabular}

The inadequacy of jitter measures to evaluate contours similarities is confirmed from the results of the accuracy measures. No significant change is observed between results before and after the alignment, while $\beta$ is approximately reduced to the half. The reliability measures obtained, as well as the reduction in $\beta$, demonstrate the occurrence of the described shifts. The occurrence of Pls is caused by some missing pulses in the real signals, while the PDAs are forced to detect pulses in those places. The measures of reliability produced by the DTW algorithm should be seen as an initial performance measure for a given PDA under evaluation.

Regarding the performance of the PDAs, there is no definitive superiority of any of the three, although "Med" seems to commit fewer errors, according to the results of reliability measures.

\section{Conclusions}

The proposed DTW procedure produced an increment in contour similarities (reduction of $\beta$ to the half) and demonstrated the occurrence of shifts in practice. The measures of performance obtained can serve as an initial indicator of the PDAs performance, and the aligned $T_{d}$ contour used for accuracy measures.

Other choices for the parameters of the DTW procedure (threshold values, number of pulses checked, etc.) should be evaluated in future works. The determination of the actual superiority of a given PDA would require the use of synthetic signals, with more precise reference contours. This can be the objective of a lengthier paper.

\section{References}

1. Hess, W.: Pitch Determination of Speech Signal: Algorithms and Devices. Springer, Berlin (1983)

2. Hess, W.J.: Pitch and Voicing Determination. In: Furui, S., Sondhi, M.M. (eds.) Advances in Speech Signal Processing. Marcel Dekker, New York (1992)

3. Titze, I.R., Liang, H.: Comparison of Fo extraction methods for high-precision voice perturbation measurements. J. Speech Hear Res. 36, 1120-1133 (1993)

4. Bagshaw, S., Hiller, M., Jack, M.A.: Enhanced pitch tracking and the processing of F0 contours for computer aided intonation teaching. In: Proc. of Eurospeech, pp. 1003-1006 (1993)

5. Titze, I.R.: Summary Statement. In: Workshop-on acoustic voice analysis. National Center of Voice and Speech, Iowa (1995) 
6. Parsa, V., Jamieson, D.G.: A comparison of high precision Fo extraction algorithms for sustained vowels. J. Speech Lang. Hear. Res. 42, 112-126 (1999)

7. Veprek, P., Scordilis, M.S.: Analysis, enhancement and evaluation of five pitch determination techniques. Speech Communication 37, 249-270 (2002)

8. de Chevigné, A., Kawahara, H.: Comparative evaluation of F0 estimation algorithms. In: Proceedings of EuroSpeech 2001, Scandinavia (2001)

9. Milenkovick, P.: Least mean squares measures of voice perturbation. J. Speech Hear. Res. 30, 529-538 (1987)

10. Boersma, P., Weenink, D.: Praat: doing phonetics by computer (2007), http: //www. fon.hum. uva.nl/praat/

11. Rabiner, L.R., Cheng, M.J., Rosemberg, A.E., McGonegal, C.A.: A comparative study of several pitch-detection algorithms. IEEE Trans. Acoust. Speech Signal Process 24, 399 417 (1976)

12. Nakatani, T., Irino, T.: Robust and accurate fundamental frequency estimation based on dominant harmonic components. J. Acoust. Soc. Am. 116, 3690-3700 (2004)

13. Shahnaz, C., Zhu, W.P., Ahmad, M.O.: Robust pitch estimation at very low SNR exploiting time and frequency domain cues. In: ICASSP 2005, pp. 389-392 (2005)

14. Moorer, J.A.: The optimum comb method of pitch period analysis of continuous digitized speech. IEEE Trans. Acoust. Speech \& Sig. Proc. 22, 330-338 (1974)

15. Deem, J.F., Manning, W.H., Knack, J.V., Matesich, J.S.: The automatic extraction of pitch perturbation using microcomputers: Some methodological considerations. J. of Speech Hear. Res. 32, 689-697 (1989)

16. Deliyski, D.D., Shaw, H.S., Evans, M.K.: Adverse effects of environmental noise on acoustic voice quality measures. J. of Voice 19, 15-28 (2005)

17. Wise, J.D., Caprio, J.R., Parks, T.W.: Maximum likelihood pitch estimation. IEEE Trans. Acoust. Speech \& Signal Processing 24, 418-423 (1976)

18. Baken, R.J.: Clinical Measurement of Speech and Voice. Singular, San Diego (1999)

19. Karnell, M.P., Hall, K.D., Landahl, K.L.: Comparison of fundamental frequency and perturbation measurements among three analysis systems. J. Voice 9, 383-393 (1995)

20. Bielamowicz, S., Kreiman, J., Gerratt, B.R., Dauer, M.S., Berke, G.S.: Comparison of voice analysis systems for perturbation measurement. J. Speech Hear Res. 39, 126-134 (1996)

21. Perry, C.K., Ingrisano, D.R., Palmer, M.A., McDonald, E.J.: Effects of environmental noise on computer-derived voice estimates from female speakers. J. Voice 14, 146-153 (2000)

22. Perry, C.K., Ingrisano, D.R., Eggleston, K.D.: The effect of noise on computer-aided measures of voice: A comparison of CSpeechSP and the Multi-Dimensional Voice Program software using the CSL 4300B module and Multi-Speech for Windows. J. Voice. 17, 12-20 (2003)

23. Medan, Y., Yair, E., Chazan, D.: Super resolution pitch determination of the speech signals. IEEE Trans. Signal Proc. 39, 40-48 (1991)

24. Kay Elemetrics Corp. Voice disorders database. Voice and Speech Laboratory Massachussets Eye and Ear Infirmary. 2 BridgeWater Lane, Lincoln Park. NJ 07035, USA (1994)

25. Ross, M.J., Shaffer, H.L., Cohen, A., Freudberg, R., Manley, H.J.: Average magnitude difference function pitch extractor. IEEE Trans. on Ac. Speech and Signal Proc. 5, 353-362 (1974) 